\title{
Erratum to: Evaluation of an ensemble of regional hydrological models in 12 large-scale river basins worldwide
}

\author{
Shaochun Huang ${ }^{1} \cdot$ Rohini Kumar $^{2}$ - Martina Flörke ${ }^{3}$. \\ Tao Yang ${ }^{4}$ - Yeshewatesfa Hundecha ${ }^{5}$ - Philipp Kraft ${ }^{6}$. \\ Chao $\mathrm{Gao}^{7}$ - Alexander Gelfan ${ }^{8} \cdot$ Stefan Liersch $^{9}$. \\ Anastasia Lobanova $^{9}$ - Michael Strauch ${ }^{2}$. \\ Floris van Ogtrop ${ }^{10}$. Julia Reinhardt ${ }^{9}$. \\ Uwe Haberlandt $^{11}$ • Valentina Krysanova ${ }^{9}$
}

\section{Erratum to: Climatic Change DOI: 10.1007/s10584-016-1841-8}

The original publication includes the following sentence in the Results section: "In general, both types of regional models, conceptual and process-based, have similar performances for

The online version of the original article can be found at http://dx.doi.org/10.1007/s10584-016-1841-8.

Shaochun Huang

shh@nve.no

Norwegian Water Resources and Energy Directorate (NVE), Oslo, Norway

2 UFZ-Helmholtz Centre for Environmental Research, Leipzig, Germany

3 Center for Environmental Systems Research, University of Kassel, Kassel, Germany

4 State Key Laboratory of Hydrology-Water Resources and Hydraulic Engineering, Center for Global Change and Water Cycle, Hohai University, Nanjing, China

5 Swedish Meteorological and Hydrological Institute, Norrköping, Sweden

6 Justus-Liebig-University Gießen, Gießen, Germany

7 College of Territorial Resources and Tourism, Anhui Normal University, Wuhu, China

8 Water Problems Institute, Russian Academy of Sciences, Moscow, Russian Federation

9 Potsdam Institute for Climate Impact Research (PIK), Potsdam, Germany

10 Centre for Carbon, Water and Food (FAE), The University of Sydney, Sydney, Australia

11 Institute of Water Resources Management, Leibniz University of Hannover, Hannover, Germany 
the 12 large-scale basins, and HBV and SWIM perform slightly better than other models in terms of monthly hydrographs, seasonal dynamics and high flows for most basins." In this erratum, we would like to clarify and stress the intent of this sentence to avoid any misunderstandings and the impression of a general conclusion about model performance.

The bold part of this sentence should not be confused with an identification of the best performing models (as may appear from this sentence). Indeed, this was neither our intention in this study nor the goal of the regional water sector modelling under the ISI-MIP project umbrella (see the editorial paper by Krysanova and Hattermann (2016) in this Special Issue). Moreover, we attempted to make clear elsewhere in the paper that an inter-comparison of the performance of individual models is not reasonable because (i) individual models were applied to a different number of river basins (varying from two to eleven), and (ii) modellers were given freedom to setup their respective models without any restrictions both technically and subjectively and could use their prior knowledge in fine tuning the models for specific basins. The modellers also had some freedom to use input data either from suggested global datasets or other sources, with the notable exception of the climate forcing data which were from the same source for all basins. Hence, on the basis of a priori knowledge and some basin-specific input data, some models may have advantages compared to others.

We would also like to stress that the focus of this study was to provide a plausible range of model skill scores of discharge derived from state-of-the-art statistical methods, simulated by different hydrological models for a set of large river basins worldwide. The ranges of model skill scores (presented in two figures and a table in the paper) should not be interpreted as a basis for ranking or identifying the best performing model(s), but rather as an indication of the ensemble range of model performances in simulating discharge under the present climate conditions. This is in alignment with our commitment to avoiding the ranking of models in this study. 